\title{
IMPORTÂNCIA DA REGIÃO ANTEROVENTRAL DO TERCEIRO VENTRÍCULO (AV3V) NO CONTROLE CARDIOVASCULAR E DO EQUILÍBRIO HIDROELETROLÍTICO
}

\author{
IMPORTANCE OF THE ANTEROVENTRAL THIRD VENTRICLE (AV3V) REGION \\ IN CARDIOVASCULAR CONTROL AND HYDROMINERAL BALANCE
}

\author{
Alexandre A Vieira ${ }^{1}$, Patrícia M De Paula², Laurival A De Luca $\mathrm{Jr}^{2}$, Débora S A Colombari², \\ Eduardo Colombari ${ }^{2}$, José V Menani ${ }^{2 *}$
}

\begin{abstract}
1Doutorando. Faculdade de Odontologia de Araraquara - UNESP. Programa de Pós-graduação em Ciências Fisiológicas. UFSCar. Convênio - UNESP/UFSCar. ${ }^{2}$ Docente. Faculdade de Odontologia de Araraquara - UNESP

CoRRESPondÊnCIA: José V. Menani. Departamento de Fisiologia e Patologia. Faculdade de Odontologia de Araraquara - UNESP

Rua Humaitá, 1680 - CEP 14.801 903, Araraquara - SP

Telefone: (16) 33016486 / FAX: (16) 33016488 / E-mail: menani@foar.unesp.br
\end{abstract}

Vieira AA, De Paula PM, De Luca Jr LA, Colombari DSA, Colombari E, Menani JV. Importância da região anteroventral do terceiro ventrículo (AV3V) no controle cardiovascular e do equilíbrio hidroeletrolítico. Medicina (Ribeirão Preto) 2006; 39 (1): 21-27.

RESUMO: A manutenção da pressão arterial em níveis normais é importante para a homeostasia do meio interno. O sistema nervoso central regulando a atividade dos eferentes autonômicos simpático e parassimpático ajusta a pressão arterial possibilitando ao animal ou ao ser humano um melhor desempenho frente a diferentes situações do cotidiano. Diferentes áreas centrais são responsáveis pelo controle das descargas autonômicas sobre o sistema cardiovascular e muitas delas também participam do controle do equilíbrio hidroeletrolítico. Uma dessas áreas é o tecido periventricular ao redor da porção anteroventral do terceiro ventrículo (região AV3V) localizado no prosencéfalo e que é uma das principais áreas centrais onde se localizam receptores da angiotensina II e osmorreceptores. A lesão da região AV3V impede o desenvolvimento de diversas formas de hipertensão experimental em ratos e dificulta o aparecimento de respostas pressoras produzidas por diversos estímulos. A lesão da região AV3V também reduz respostas dipsogênicas induzidas pela angiotensina II, estimulação colinérgica central, privação hídrica e aumento de osmolaridade plasmática, a secreção do peptídeo natriurético atrial produzida pela expansão de volume e a excreção renal de sódio produzida pela estimulação colinérgica central. Evidências mais recentes também sugerem uma participação da região AV3V nas respostas pressoras produzidas pela ativação de mecanismos bulbares.

Descritores: Sistema Nervoso Simpático. Angiotensina. Hipertensão. Hipotálamo. Glutamato.

\section{1- INTRODUÇÃO}

O bom funcionamento do sistema cardiovascular e, em particular, a manutenção de uma pressão arterial em níveis normais é importante para uma oferta adequada de suprimentos necessários à sobrevivência das células. Mesmo que o sistema cardiovascular pudesse desenvolver seu papel na ausência do sistema nervoso central (SNC), é este último que detém os mecanismos refinados de ajustes capazes de regular e manter em níveis adequados a pressão arterial, a resistência periférica, a frequência cardíaca e o débito cardíaco. 
O SNC ajusta a atividade dos eferentes autonômicos simpático e parassimpático, o que juntamente com componentes hormonais, possibilita ao animal ou ser humano um melhor desempenho frente a diferentes situações cotidianas. Além disso, existe uma íntima correlação entre o funcionamento do sistema cardiovascular e o balanço dos líquidos corporais, pois mudanças em um deles geralmente implica em ajustes no outro.

A manutenção adequada do equilíbrio hidroeletrolítico depende da presença de um íon importante, cuja quantidade está diretamente ligada ao volume plasmático, o sódio. O controle do balanço entre o ganho (através da ingestão) e perda (através da excreção) de água e sódio no organismo evita modificações no volume de líquido extracelular que poderiam refletir de modo negativo nas funções cardiovasculares e eventualmente na função celular. Modificações do volume plasmático alteram a atividade dos receptores periféricos (pressorreceptores e receptores cardiopulmonares) que promovem ajustes nos mecanismos neuroendócrinos de controle da pressão arterial.

Impulsos nervosos do sistema nervoso simpático e parassimpático são importantes para a manutenção do tônus dos leitos vasculares periféricos, da atividade cardíaca e conseqüentemente da pressão arterial. Mas a mesma atividade simpática é importante para o controle da função renal que é responsável pela manutenção do volume e da osmolalidade plasmática, que podem influenciar a função cardiovascular. Embora o controle básico cardiovascular seja mantido por áreas pertencentes ao tronco cerebral, outras áreas cerebrais também estão envolvidas com o controle cardiovascular. Uma dessas áreas está localizada no prosencéfalo e é constituída pelo tecido periventricular ao redor da porção anteroventral do terceiro ventrículo (região AV3V).

\section{2- A REGIÃo AV3V}

A região $\mathrm{AV} 3 \mathrm{~V}$ tem componentes sensíveis a alterações na osmolaridade plasmática e concentração de hormônios circulantes, como o peptídeo angiotensina II (ANG II). Além de ser rica em receptores para a ANG II, a região AV3V também possui receptores para acetilcolina e glutamato ${ }^{1 / 6} \mathrm{e}$ várias conexões intrínsecas e extrínsecas com circuitos que promovem ajustes cardiovasculares e no equilíbrio hidroeletrolítico $^{7 / 10}$.

A região $\mathrm{AV} 3 \mathrm{~V}$ é constituída pelo órgão vasculoso da lâmina terminal (OVLT), altamente vascularizado e livre de barreira hemato-encefálica localizado na parede anterior do terceiro ventrículo, o tecido periventricular da região preóptica e da porção anterior da área hipotalâmica anterior e pela parte ventral do núcleo preóptico mediano (Figura 1A,B) ${ }^{7 / 9}$. A definição explícita da região AV3V tem origem em estudos com lesão eletrolítica em ratos ${ }^{7 / 9}$ e é importante ressaltar que ela exclui a área preóptica medial, o núcleo hipotalâmico anterior e o núcleo paraventricular do hipotálamo (PVN). Entretanto, estudos pioneiros com injeções hiperosmóticas em diferentes áreas prosen-
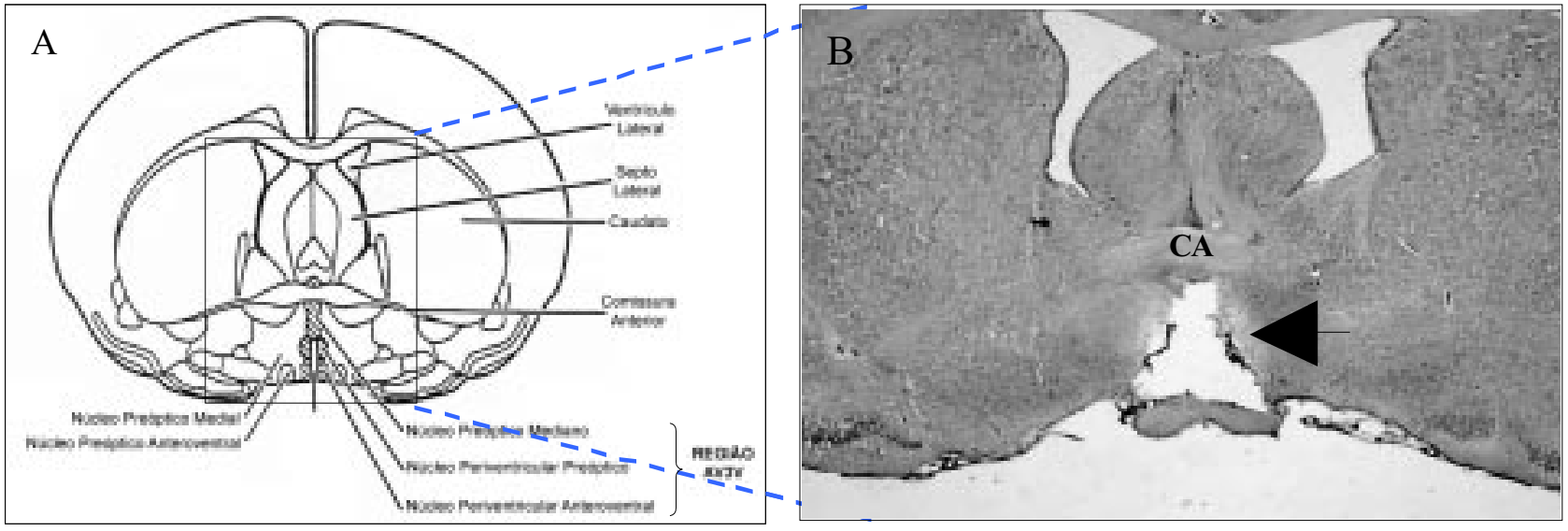

Figura 1: A: Diagrama ilustrando os grupos celulares que formam a região AV3V. Note que OVLT fica imediatamente rostral ao núcleo periventricular anteroventral e é considerado parte da região AV3V. B: Fotomicrografia de um corte histológico do cérebro de um animal, indicando (seta) a área da lesão da região AV3V. Comissura anterior (CA). 
cefálicas já sugeriam a importância dessa região para o controle da sede ${ }^{11}$. Além da participação no controle cardiovascular e do equilíbrio hidroeletrolítico, a região AV3V também está envolvida no controle da reprodução, comportamento maternal, termorregulação e modulação dos sistemas imunes e neuroendócri$\operatorname{nos}^{12,17}$.

Estimulação elétrica da região $\mathrm{AV} 3 \mathrm{~V}$ produz vasodilatação dos membros e vasoconstrição renal e mesentérica associadas a respostas depressoras e bradicardia. As respostas vasculares são dependentes de inervação simpática direta ${ }^{7,18} \mathrm{e}$, em parte, também dependente das catecolaminas produzidas pela adrenal ${ }^{19}$.

\section{3- IMPORTÂNCIA DA REGIÃO AV3V - EFEI- TOS DA LESÃO ELETROLÍTICA}

A lesão eletrolítica da região AV3V impede o desenvolvimento de diversos modelos de hipertensão experimental em ratos, com exceção da hipertensão espontânea ${ }^{7 / 9,20,21}$. As respostas pressoras, assim como a ingestão de água induzida por ANG II periférica ou central são bloqueadas pela lesão da região $\mathrm{AV} 3 \mathrm{~V}^{7,9}$. Esta mesma lesão também atenua o aumento da pressão arterial, ingestão de água, natriurese e caliurese induzida pela injeção intracerebroventricular (icv) de carbacol (agonista colinérgico) ${ }^{4}$ e mesmo ativação colinérgica de diversas áreas prosencefálicas, como área septal medial (ASM), órgão subfornical (OSF), hipotálamo ventro-medial (HVM) e área preóptica lateral (APL) $)^{22 / 25}$, ou caudais como locus coeruleus ${ }^{26}$. A lesão aguda da região $\mathrm{AV} 3 \mathrm{~V}$ também reduz a salivação induzida pela pilocarpina (intraperitoneal ou icv), o que tem contribuído para suportar a sugestão de que parte do efeito sialogogo deste agonista colinérgico depende também de uma ação central do mes$\mathrm{mo}^{27,28}$. Embora a lesão da região $\mathrm{AV} 3 \mathrm{~V}$ prejudique respostas pressoras de diversas origens, os animais com lesão aguda da região AV3V podem apresentar um pequeno aumento da pressão arterial acompanhada de taquicardia nos primeiros dias após a lesão ${ }^{21}$.

Um dos marcantes efeitos que ocorre nos primeiros cinco dias após a lesão da região $\mathrm{AV} 3 \mathrm{~V}$, é a abrupta abolição na ingestão diária de água ${ }^{29}$. É importante ressaltar que a interrupção na ingestão de água não é devida a comprometimento motor, pois os animais lesados locomovem-se normalmente em busca de soluções palatáveis, ingerem alimento e exibem comportamentos de alerta parecidos com os de animais com lesão fictícia.
A perda de água pela urina também é aumentada, pois os animais com lesão da região AV3V apresentam comprometimento na liberação de vasopres$\operatorname{sina}^{30}$. A menor liberação de vasopressina, pode ser devida ao comprometimento de uma via que projetase da região $\mathrm{AV} 3 \mathrm{~V}$ diretamente para o $\mathrm{PVN}$, levando à perda de um sinal facilitatório para o PVN que acabaria resultando em atenuação da liberação de vasopressina. Como conseqüência da adipsia e do aumento da excreção urinária, os animais com lesão da região AV3V podem perder até $25 \%$ de seu peso corporal nos primeiros 3 dias após a lesão, (Tabela I). Paralelamente, os ratos com lesão da região AV3V apresentam aumento da osmolalidade plasmática ${ }^{31}$. Por isso, é recomendável que os animais submetidos à lesão da região $\mathrm{AV} 3 \mathrm{~V}$ sejam hidratados por meio de infusão intra-gástrica (gavagem) ou pelo oferecimento de soluções palatáveis, sacarose $10 \%$ por exemplo, para ingestão. A hidratação forçada por pelo menos uma semana é necessária para que se evite a morte dos animais até que eles superem o período mais crítico de adipsia voltando a ingerir água voluntariamente. Entretanto, a retirada das soluções contendo sacarose $10 \%$ não deve ser abrupta, ou seja, a melhor maneira de se garantir um retorno da ingestão de água, reduzindo ao máximo uma possível desidratação, é pela diminuição diária da concentração de açúcar na solução até a substituição completa da sacarose pela água.

Cronicamente após a lesão da região $\mathrm{AV} 3 \mathrm{~V}$ a ingestão diária de água retorna aos valores normais, mas está atenuada a ingestão de água induzida por privação hídrica e abolida a ingestão de água induzida por desidratação celular. Além disso, os animais também exibem hiperosmolalidade plasmática ${ }^{31}$, além de outros efeitos listados na Tabela I. A recuperação de algumas respostas em animais com lesão crônica da região AV3V pode ser devida a uma possível plasticidade cerebral com alterações e adaptações nas funções de algumas áreas cerebrais.

A lesão da região $\mathrm{AV} 3 \mathrm{~V}$ também reduz a natriurese que ocorre durante a privação hídrica e os níveis plasmáticos de peptídeo natriurético atrial (ANP) em condições de repouso, além de prejudicar o aumento da secreção de ANP induzida pela expansão de volume $\mathrm{e}^{32 / 36}$. Os impulsos nervosos que ascendem dos barorreceptores (aórticos e carotídeos), influenciam momento a momento a região $\mathrm{AV} 3 \mathrm{~V}$ para o controle da liberação de ANP via sistemas neuronais hipotalâmicos ${ }^{35}$. 
Tabela I: Efeitos da lesão da região AV3V

\section{Efeitos Agudos}

- Adipsia;

- Diminuição da secreção de vasopressina;

- Diminuição da resposta pressora e ingestão de água induzidas pela ativação angiotensinérgica central;

- Diminuição da resposta pressora, ingestão de água e natriurese induzidas pela ativação colinérgica central;

- Bloqueio da salivação e da resposta pressora induzidas pelo agonista colinérgico pilocarpina ip;

- Atenuação da resposta pressora produzida pela oclusão bilateral das carótidas;

- Bloqueio da resposta simpato-excitatória produzida pela injeção de glutamato no NTS;

- Atenuação da resposta simpato-excitatória produzida pela injeção de glutamato na área RVL do bulbo;

- Severa perda de peso e se não tratada, debilitação e morte devido a desidratação.

\section{Efeitos Crônicos}

- Redução do peso corporal;

- Atenuação da ingestão de água induzida por privação hídrica, estimulação colinérgica central, isoproterenol sc, ligadura

- da veia cava ou polietilenoglicol sc;

- Abolição da ingestão de água induzida por desidratação celular e angiotensina II;

- Redução do apetite ao sódio induzido por depleção de sódio ou dependente de ANG II;

- Redução da secreção de vasopressina após salina hipertônica e ANG II;

- Redução da natriurese e hipernatremia;

- Redução da resposta pressora induzida pela salina hipertônica, estimulação colinérgica ou angiotensinérgica central ou oclusão bilateral das carótidas;

- Bloqueio da resposta simpato-excitatória produzida pela injeção de glutamato no NTS;

- Atenuação da resposta simpato-excitatória produzida pela injeção de glutamato na área RVL do bulbo.

ip = intraperitoneal; sc = subcutâneo; NTS =núcleo do trato solitário; área RVL = área rostroventrolateral; ANG II = angiotensina II

\section{4- IMPORTÂNCIA DA REGIÃO AV3V PARA RESPOSTAS CARDIOVASCULARES PRO- DUZIDAS PELA ATIVAÇÃO DE ÁREAS DO TRONCO CEREBRAL}

Recentes estudos de nosso laboratório ${ }^{37,38}$ sugerem que a região AV3V pode modular a atividade neural simpática por influenciar mecanismos que são ativados pelas áreas do tronco cerebral, como por exemplo, o núcleo do trato solitário (NTS) e a área rostroventro lateral do bulbo (RVL) que fazem parte do circuito bulbar envolvido no controle cardiovascular. O NTS tem um papel importante na regulação cardiovascular, pois é o sítio onde as aferências dos pressorreceptores, quimiorreceptores e receptores cardiopulmonares fazem a primeira sinapse no $\mathrm{SNC}^{39,40}$. A área RVL por sua vez é a principal área central envolvida na ativação dos neurônios pré-ganglionares simpáticos e, portanto, responsável pela geração e manutenção do tônus vasomotor simpático ${ }^{41 / 45}$. $\mathrm{O}$ aminoácido excitatório glutamato é considerado o principal neurotransmissor liberado pelas aferências dos pressorreceptores ${ }^{46 / 50}$ e quimiorreceptores periféri$\cos ^{51,52,53}$ no NTS, bem como na área RVL ${ }^{54}$.

A injeção de glutamato tanto no NTS, quanto na área RVL de ratos acordados produz resposta pressora e aumento da atividade simpática ${ }^{37,38,49,50}$. A lesão aguda (1 dia) e crônica (15 dias) da região AV3V abole a resposta pressora do glutamato injetado no NTS e atenua a resposta pressora do glutamato injetado na área $\mathrm{RVL}^{37,38}$. Esses resultados sugerem o envolvimento de mecanismos dependentes de áreas prosencefálicas, em especial da região $\mathrm{AV} 3 \mathrm{~V}$, nas respostas pressoras induzidas pelo glutamato atuando em 
área bulbares. Por outro lado, apesar da lesão da região AV3V (aguda ou crônica) influenciar fortemente as respostas pressoras produzidas pela ativação glutamatérgica de áreas bulbares, não se observa o comprometimento da atividade quimiorreflexa e barorreflexa $^{37,55}$, bem como na reatividade vascular ${ }^{56}$.

Projeções diretas ou indiretas da região AV3V para diversas áreas do tronco cerebral, incluindo NTS e área RVL já foram descritas ${ }^{57,58,59}$. Os efeitos da lesão da região AV3V reduzindo ou abolindo respostas pressoras de diversas origens e prejudicando o desenvolvimento de diversas formas de hipertensão coloca essa área como uma importante área integradora e com um papel importante para respostas cardiovasculares, em especial respostas hipertensoras. O circuito bulbar do qual fazem parte o NTS e o RVL é fundamental para a regulação cardiovascular, mas a resposta final pode depender da participação de um circuito central muito mais complexo onde áreas prosencefálicas podem modular a resposta à estimulação de uma determinada área bulbar.

Considerando-se resultados dos estudos já realizados, é muito clara a forte influência que a região
AV3V têm sobre a atividade simpática. Conexões diretas entre a região $\mathrm{AV} 3 \mathrm{~V}$ e as áreas prosencefálicas envolvidas no controle cardiovascular como o OSF, HVM, ASM e APL podem explicar parte da influência da região AV3V no controle da atividade simpática 9,59/62. Além disso, conexões entre a região AV3V e o $\mathrm{PVN}$ e a região $\mathrm{AV} 3 \mathrm{~V}$ e áreas do tronco cerebral, além de projeções da região AV3V diretas para a IML já foram mostradas ${ }^{58,59}$. Portanto, seja facilitando a ativação do sistema simpático ou a ativação e/ou ação de mecanismos hormonais, a região AV3V tem marcante envolvimento com a regulação cardiovascular.

\section{AGRADECIMENTOS}

Nossos agradecimentos aos colegas citados nas referências que tiveram grande contribuição para o entendimento do papel da região $\mathrm{AV} 3 \mathrm{~V}$ no controle cardiovascular e hidro-salino. A pesquisa conduzida pelos autores teve apoio da Fundação de Amparo à Pesquisa do Estado de São Paulo (FAPESP) e Conselho Nacional de Desenvolvimentos Científico e Tecnológico (CNPq)/PRONEX

Vieira AA, De Paula PM, De Luca Jr LA, Colombari DSA, ColombariE, Menani JV. Importance of the anteroventral third ventricle (AV3V) region in cardiovascular control and hydromineral balance. Medicina (Ribeirão Preto) 2006; 39 (1): 21-27.

ABSTRACT: The maintenance of the arterial pressure in normal levels is important for the homeostasis of body fluids. The central nervous system regulating sympathetic and parasympathetic autonomic efferent can adjust arterial pressure which allows animals or human to face different daily activities with the best performance. Different central areas are responsible for the control of autonomic discharges to cardiovascular system and many of them are also involved in the control of fluid electrolyte balance. One of these areas is the tissue surrounding the anteroventral third ventricle (AV3V region) localized in the forebrain and a main central site for angiotensin II receptors and osmoreceptors. The AV3V lesions impair the development of many models of experimental hypertension in rats and the pressor responses to different stimuli. Lesions of the $\mathrm{AV} 3 \mathrm{~V}$ region also reduce dipsogenic responses to angiotensin II, central cholinergic activation, water deprivation and increase in plasma osmolarity, atrial natriuretic peptide secretion produced by body fluid expansion and the increase in renal excretion to central cholinergic activation. Recent evidence also suggests the participation of AV3V region in pressor responses produced by the activation of medullary mechanisms.

Keywords: Sympathetic Nervous System. Angiotensin. Hypertension. Hypothalamus. Glutamate. 


\section{REFERÊNCIAS}

1 - Bealer SL. Sistemic angiotensin II alters intrinsic heart rate through central mechanisms. Brain Res Bull 2002; 58: 61-55.

2 - Lenkey Z, Corvol P, Llorens-Cortes $C$. The angiotensin receptor subtype AT1A predominates in rat forebrain areas involved in blood pressure, body fluid homeostasis and neuroendocrine control. Brain Res Mol Brain Res 1995; 30: 53-60.

3 - Morris M McCann SM, Orias R. Role of transmitters in mediating hypothalamic control of electolyte excretion. Can J Physiol Pharmacol 1977; 55:1143-54.

4 - Menani JV, Saad WA, Camargo LA, Renzi A, De Luca Junior $\mathrm{LA}$, Colombari E. The anteroventral third ventricle (AV3V) region is essential for pressor, dipsogenic and natriuretic responses to central carbachol. Neurosci Lett 1990; 113: 339-44.

5 - Cotman CW, Monaghan DT, Ottersen OP, Storm-Mathisen J. Anatomical organization of excitatory amino acid receptors and their pathways. Trends Neurosci 1987; 10:273-80.

6 - Yamagushi K, Watanabe K. Anteroventral third ventricular $\mathrm{N}$-methyl-D-aspartate receptors, but not metabotropic glutamate receptors are involved in hemorrhagic AVP secretion. Brain Res Bull 2005; 66:59-69.

7 - Brody MJ, Fink GD, Buggy T, Haywood JR, Gordon FJ, Johnson AK. The role of the anteroventral third ventricle (AV3V) region in experimental hypertension. Circ Res1978; 43:1-13.

8 - Brody MJ, Faber JE, Mangiapane ML, Porter JP. The central nervous system and prevention of hypertension. In: De Jong W, ed. Experimental and genetic models of hypertension. Amsterdan: W. Elsevier; 1984. p. 474-94. (Handbook of Hypertension, Vol. 4).

9 - Brody MJ, Johnson AK. Role of the anteroventral third ventricle region in fluid and electrolyte balance, arterial pressure regulation and hypertension. In: Martini L, Ganong WF, eds. Frontiers in neuroendocrinology. New York: Raven Press; 1980. p. 249-92.

10 - De Luca JR LA, Menani JV. Preoptic-periventricular tissue (AV3V): central cholinergic-induced hydromineral and cardiovascular responses, and salt intake. Rev Bras Biol 1996; 56 (Supl. 1):233-8.

11 - Andersson B. The effect of injections of hypertonic $\mathrm{NaCl}$ solutions into differents parts of hypothalamus of goats. Acta Physiol Scand 1953; 28: 188-201.

12 - Johnson AK, Gross PM. Sensory circumventricular organs and brain homeostatic pathways. FASEB J 1993; 7:678-86.

13 - Numan M. A neural circuitry analysis of maternal behavior in the rat. Acta Paediatr 1994 (Suppl 397): 19-28.

14 - Boulant JA. Hypothalamic neurons regulating body temperature. In: Fregly MJ, Blatteis CM, eds. APS Handbook of physiology. New York: Oxford University Press; 1996. p. 105-26. Section 4: Environmental physiology.

15 - Elmquist JK, Scammell TE, Saper CB. Mechanisms of CNS response to systemic immune challenge: the febrile response. Trends Neurosci 1997; 20:565-70.
16 - Hori T, Katafuchi T, Take S, Shimizu N. Neuroimmunomodulatory actions of hypothalamic interferon-alpha. Neuroimmunomodulation 1998; 5:172-7.

17 - Simerly RB. Organization and regulation of sexually dimorphic neuroendocrine pathways. Behav Brain Res 1998; 92:195-203.

18 - Fink GD, Buggy J, Haywood JR, Johnson AK, Brody MJ. Hemodynamic responses to electrical stimulation of areas of rat forebrain containing angiotensin on osmosensitive sites. Am J Physiol 1978; 235: H445-51.

19 - Knuepfer MM, Johnson AK, Brody MJ. Vasomotor projections from the anteroventral third ventricle (AV3V) region. Am J Physiol 1984; 247:H139-45.

20 - Menani JV, Bedran De Castro MT, Krieger EM. Influence of the anteroventral third ventricle region and sinoaortic denervation on the pressor response to carotid occlusion. Hypertension 1988; 11: I-178-81.

21 - Menani JV, Machado BH, Krieger EM, Salgado HC. Tachycardia during the onset of one kidney one-clip renal hypertension: role of renin-angiotensin system and AV3V tissue. Brain Res 1988; 446: 295-302.

22 - Colombari E, Saad WA, Camargo LA, Renzi A, De Luca Junior LA, Menani JV. AV3V lesion suppresses the pressor, dipsogenic and natriuretic responses to cholinergic activation of the septal area in rats. Brain Res 1992; 572:172-5.

23 - Colombari DS, Saad WA, Camargo LA, Renzi A, De Luca Junior LA, Colombari E, Menani JV. AV3V lesion impairs responses induced by cholinergic activation of SFO in rats. Am J Physiol 1992; 263: R1277-83.

24 - Valladão AS, Saad WA, Camargo LA, Renzi A, De Luca Junior LA, Menani JV. AV3V lesion reduces the pressor, dipsogenic, and natriuretic responses to ventromedial hypothalamus activation Brain Res Bull 1992; 28: 909-14.

25 - Goncalves PC, Alves MB, Silveira JE, Saad WA, Camargo LA, Renzi A, De Luca Junior LA, Menani JV. Effect of AV3V lesion on the cardiovascular, fluid, and electrolytic changes induced by activation of the lateral preoptic area. Physiol Behav 1992; 52:173-7.

26 - De Luca Jr LA, Franci CR, Saad WA, Camargo LAA, AntunesRodrigues J. Natriuresis, not seizures, induced by cholinergic stimulation of the locus coeruleus is affected by forebrain lesions and water deprivation. Brain Res Bull 1991; 26:203-10.

27 - Takakura ACT, Moreira TS, De Luca Jr LA, Renzi A, Menani JV, Colombari E. Effects of AV3V lesion on pilocarpine-induced pressor response and salivary gland vasodilation. Brain Res 2005; 1055:111-21.

28 - Renzi A, Colombari E, Mattos Filho TR, Silveira JEN, Saad WA, Camargo LAA, De Luca Jr LA, Deróbio JG, Menani JV. Involvment of the central nervous system in the salivary secretion induced by pilocarpine in rats. J Dent Res 1993; 72:1481-4.

29 - Johnson AK, Buggy J. Periventricular preoptic-hypothalamus is vital for thirst and normal water economy. Am J Physiol 1978; 234:R122-9.

30 - Bealer SL, Phillips MI, Johnson AK, Schmid PG. Anteroventral third ventricle lesions reduce antidiuretic responses to angiotensin II. Am J Physiol 1979; 236: E610-5. 
31 - Buggy J, Johnson AK. Preoptic-hypothalamic periventricular lesions: thirst deficits and hypernatremia. Am J Physiol Regul Integr Comp Physiol 1977; 233: R44-52.

32 - Buggy J, Bealer SL. Physiological regulation by the AV3V region. In: Gross PM, ed. Circumventricular organs and body fluids. Boca Raton: CRC Press; 1987. Vol. 1. p.171-90.

33 - Mckinley M. Common aspects of the cerebral regulation of thirst and renal sodium excretion. Kidney Int 1992; 41: S102-6.

34 - Baldissera S, Menani JV, Dos Santos LF, Favaretto AL, Gutkowska J, Turrin MQ, Mccann SM, Antunes-Rodrigues J. Role of the hypothalamus in the control of atrial natriuretic peptide release. Proc Natl Acad Sci USA 1989; 86(23):9621-5.

35 - Antunes-Rodrigues J, Favaretto AL, Gutkowska J, Mccann $\mathrm{SM}$. The neuroendocrine control of atrial natriuretic peptide release. Mol Psychiatry 1997; 2:359-67.

36 - Antunes-Rodrigues J, Ramalho MJ, Reis LC, Menani JV, Turrin MQ, Gutkowska J, Mccann SM. Lesions of the hypothalamus and pituitary inhibit volume-expansion-induced release of atrial natriuretic peptide. Proc Natl Acad Sci USA 1991; 88(7): 2956-60.

37 - Vieira AA, Colombari E, De Luca LA JR, De Almeida, Colombari DS, Menani JV. Cardiovascular responses to microinjection of L-glutamate into the NTS in AV3V-lesioned rats. Brain Res 2004; 1025:106-12.

38 - Vieira AA, De Luca LA JR, Colombari E, De Almeida Colombari DS, Menani JV. AV3V lesion impair the pressor response to glutamate into the rostral ventrolateral medulla. Program Number 922.6. Abstract Viewer/Itinerary Planner. Washington DC: Society for Neuroscience, 2003. (Abstract).

39 - Crill WE, Reis DJ. Distribution of carotid sinus and depressor nerves in the cat brain stem. Am J Physiol 1968; 214 (2): 269-76.

40- Mifflin SW. Arterial chemoreceptors input to nucleus tractus solitarius. Am J Physiol 1992; 263: R368-75.

41- Ross CA, Ruggiero DA, Park DH, Joh TH, Sved AF, FernandezPardal J, Saavedra JM, Reis DJ. Tonic vasomotor control by the rostral ventrolateral medulla: Effect of electrical or chemical stimulation of the area containing $\mathrm{C} 1$ adrenaline neurons on arterial pressure, heart rate and plasma cathecolamines and vasopressin. J Neurosci 1984; 4:474-94.

42 - Guertzeinstein PG, Silver A. Fall in blood pressure produced from discrete regions of the ventral surface of the medulla by glycine and lesions. J Physiol 1974; 242:489-503.

43 - Guyenet PG, Haselton JR, Sun MK. Sympathoexcitatory neurons of the rostroventrolateral medulla and the origin of the sympathetic vasomotor tone. Prog Brain Res 1989; 81: 105-16.

44- Morrinson SF, Reis DJ. Responses of sympathetic preganglionic neurons to rostral ventrolateral medullary stimulation. Am J Physiol 1991; 261:R1247-56.

45-Chalmers J, Pilowsky P. Brainstem and bulbospinal neurotransmitter systems in the control of blood pressure. J Hypertension 9:675-94, 1991.

46-Talman WT, Perrone MH, Reis DJ. Evidence for L-glutamate as the neurotransmitter of baroreceptor afferent nerve fibers. Science1980; 209:813-5.
47-Talman WT. Kynurenic acid microinjected into the nucleus tractus solitarius of rat blocks the arterial baroreflex but not responses to glutamate. Neurosc Lett 1989; 102:247-52.

48-Gordon FJ, Talman WT. Role of excitatory amino acids and their receptors in bulbospinal control of cardiovascular function. In: Kunos G, Ciriello J, eds. Central neural nechanisms in cardiovascular regulation. Boston: Birkhauser; 1992. p. 209-25.

49 - Colombari E, Bonagamba LGH, Machado BH. Mechanisms of pressor and bradycardic responses to L-glutamate microinjected into the NTS of conscious rats. Am J Physiol 1994; 266:R730-8.

50 - Machado BH, Bonagamba LGH. Microinjection of L-glutamate into the nucleus tractus solitarii increases arterial pressure in conscious rats. Brain Res 1992; 576:131-8.

51- Haibara AS, Colombari E, Chianca Jr DA, Bonagamba LGH, Machado $\mathrm{BH}$. NMDA receptors in NTS are involved in bradycardic but not in pressor response of chemoreflex. Am J Physiol 1995; 269:H1421-7.

52-Vardhan A, Kachroo A, Sapru HN. Excitatory amino acid receptor in commissural nucleus of the NTS mediate carotid chemoreceptor responses. Am J Physiol 1993; 264:R41-50.

53-Zhang W, Mifflin SW. Excitatory amino acid receptors within NTS mediate arterial chemoreceptor reflexes in rats. Am J Physiol 1993; 265:H770-3.

54-Sved AF, Gordon FJ. Amino acids as central neurotransmitters in the baroreceptors reflex pathway. News Physiol Sci 1994; 9: 243-6.

55-Bealer SL. Preoptic recess ablation selectively increases baroreflex sensitivity to angiotensin II in conscious rats. Peptides 1995; 16:1197-201.

56-Whalen EJ, Beltz TG, Lewis SJ, Johnson AK. AV3V lesions attenuate the cardiovascular responses produced by bloodborne excitatory amino acid analogs. Am J Physiol Heart Circ Physiol 1999; 276:H1409-15.

57-Saper CB, Reis DJ, Joh T. Medullary catecholamine inputs to the anteroventral third ventricular cardiovascular regulatory region in the rat. Neurosc Lett 1983; 42:285- 91.

58-Ricardo JA, Koh ET. Anatomical evidence of direct projections from the nucleus of the solitary tract to the hypothalamus, amygdala, and other forebrain structures in the rat. Brain Res 1978; 153:1-26.

59- Westerhaus MJ, Loewy AD. Sympathetic-related neurons in the preóptica region of the rat identified by viral transneuronal labeling. J Comp Neurol 1999; 414:361-78.

60-Miselis RR. The efferent projections of the subfornical organ of the rat: a circumventricular organ within a neural network subserving water balance. Brain Res 1981; 230:1-23.

61-Johnson AK, Buggy J, Fink GD, Brody MJ. Prevention of renal hypertension and of the central pressor effect of angiotensin by ventromedial hypothalamic ablation. Brain Res 1981; 205:255-64.

62 -Buggy J, Johnson AK. Angiotensin-induced thirst: effects of third ventricle obstruction and periventricular ablation. Brain Res 1978;149:117-28. 